\title{
Tracking water and hydroxyl species at nanomaterial surfaces and interfaces
}

Katharine Page ${ }^{a}$

a Neutron Scattering Division, Oak Ridge National Laboratory, 1 Bethel Valley Road, Oak Ridge, Tennessee 37831, USA, pagekl@ornl.gov

Neutron scattering techniques are widely known to reveal the locations, populations, and mobilities of hydrogen (and deuterium) atoms across materials classes. We will present our work following hydrogenbearing species at the surfaces and interfaces of nanostructured materials with neutron pair distribution function (PDF) studies and complementary techniques. For example, we will present the local and longrange structure of synthetic layered $\delta-\mathrm{MnO}_{2}$, from formation, through dehydration, and through phase transformation. Models incorporating stacking disorder and interlayer/surface chemistry reveal that water molecules and hydrogen bonding play key roles in maintaining long range order, while Mn vacancies within the $\mathrm{MnO}_{2}$ layers promote the intercalation of $\mathrm{Cu}^{2+}$ into interlayer spaces, forming strongly distorted interfacial octahedra.[1] These characteristics are shown to have marked influences on the charge/discharge capacity and cycling abilities of materials studied. We will additionally demonstrate abilities to follow the hydration and parasitic hydroxide formation at the surfaces of working magnesium oxide catalysts, as well as the nature of several dehydration-driven phase transformations. The sensitivities of the neutron probe to hydrogen and the PDF approach to local atomic structure combine to provides rich structural insights into traditionally challenging problems.
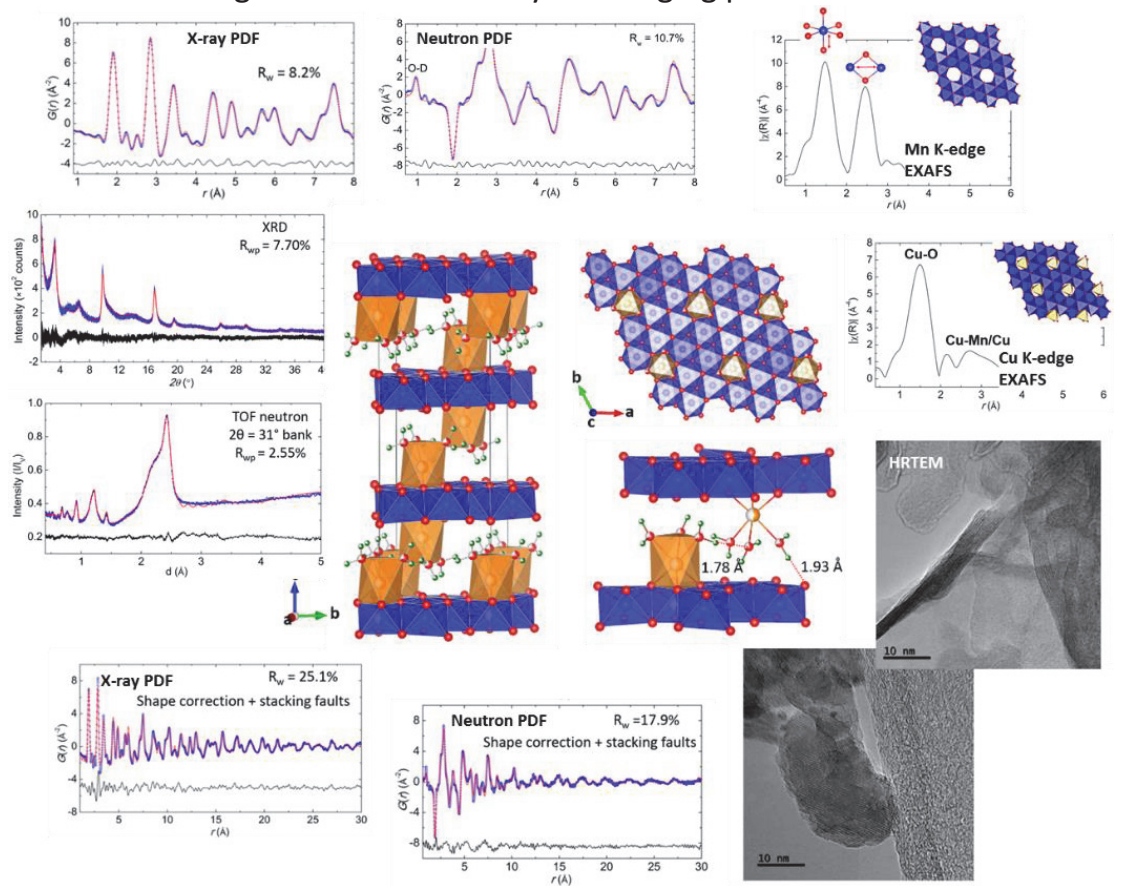

Figure 1. Comprehensive structural characterization of a heavily stacking faulted $\mathrm{Cu}$-rich $\delta-\mathrm{MnO}_{2}$ nanoflower. Local x-ray and neutron PDF, Cu K-edge and Mn K-edge EXAFS studies enable determination of the layer motif and the identification and quantification of both interlayer $\mathrm{Cu}^{2+}$ and $\mathrm{H}_{2} \mathrm{O}$, while HRTEM, intermediate-range PDF, and $\mathrm{x}$-ray and neutron diffraction approaches enable the determination of average stacking sequences and (average) nanoparticle dimensions.

[1] Liu J., Yu L., Hu E., Guiton B.S., Yang X.Q., Page K. (2018), Inorganic Chemistry, 57, 6873-6882. 